\title{
Discrimination of SNPs in GC-rich regions using a modified hydrolysis probe chemistry protocol
}

\author{
Alireza Mowjoodi ${ }^{1}$, Tara A. Paton ${ }^{1}$, and Stephen W. Scherer ${ }^{1,2,3}$ \\ 'The Centre for Applied Genomics, The Hospital for Sick Children, \\ Toronto, Ontario, Canada, ${ }^{2}$ McLaughlin Centre, University of Toronto, \\ Toronto, Ontario, Canada, and ${ }^{3}$ Department of Molecular Genetics, \\ University of Toronto, Toronto, Ontario, Canada.
}

BioTechniques 57:313-316 (December 2014) doi 10.2144/000114240

Keywords: TaqMan; SNP genotyping; allelic discrimination; 5'-nuclease; GC-rich; SNP

Allelic discrimination using TaqMan 5'-nuclease assay chemistry has been in routine use for many years, and the catalog of Life Technologies' predesigned SNP genotyping assays now exceeds 4 million entries. However, predesigned assays are often not available for genomic regions with a high GC content, nor can an assay necessarily be designed in this type of region using the manufacturer's design pipelines. Additionally, when an assay is available, the performance can be poor when using standard protocols. Here we report a modified allelic discrimination protocol for variants that reside in extremely GC-rich (GC > 75\%) regions. The approach resolves fluorescent signal from reference and variant alleles, allowing all samples to be successfully assigned a genotype call. This protocol modification adds an extra step to the standard workflow, but the increased time is a productive compromise to generate high-quality data.

The demand for targeted SNP genotyping continues to increase, in particular as next-generation sequencing technologies generate multitudes of new variants whose allelic architecture often need to be assessed in families and larger cohorts. Indeed, variants of interest derived from small whole-genome or whole-exome sequencing studies (from humans or other species) can be interrogated using high-throughput targeted SNP genotyping approaches such as hydrolysis probe chemistry, single-base extension, or other PCR-based methods. However, variants in regulatory regions, first exons and introns of genes, and some other genomic elements can be more difficult to study due to the high GC content of their surrounding sequences $(1,2)$. PCR-based assays in $\mathrm{GC}$-rich regions are challenging because of the formation of thermostable structures, resulting in incomplete denaturation of genomic template or difficulties with primer annealing $(3,4)$. The human genome reference assembly (GRC37/hg19) contains more than 300,000 SNPs in regions with $\geq 75 \%$ GC content (dbSNP137; calculated on $15 \mathrm{bp}$ of flanking sequence), thus leaving a large number of potentially interesting variants seemingly inaccessible to study. These segments of the genome may also be hotspots for mutation, but they are underrepresented in databases because of the refractory nature of detecting them.

TaqMan SNP genotyping assays (Life Technologies, Carlsbad, CA) are routinely used for allelic discrimination because of the convenience of a premixed primer and probe formulation combined with a standardized protocol that requires little optimization $(5,6)$. For SNPs that lie in GC-rich or repetitive regions, however, there is often no predesigned assay available from the Life Technologies catalog, nor can an assay be designed for these regions using the vendor's Assay Design Pipeline. In instances when an assay is available (whether catalog or user designed), the performance using the manufacturer's protocol can be poor, in that reference and variant allele probe signals cannot be distinguished (lower left quadrant, Figure 1A).

To overcome this problem, we developed a two-stage approach in which a pre-PCR of the target region is performed, followed by the standard TaqMan genotyping protocol using these PCR products as the input template. We hypothesized that the first PCR step would increase the amount of target template available for the standard assay, and the small product would denature more easily and be less likely to form secondary structures relative to the target in its genomic DNA context. This would then allow the probes to more efficiently hybridize to the SNP region, generating higher overall signal.

To evaluate this method, 3 SNP variants in regions of high $\mathrm{GC}$ content (67\%-94\%) were selected from wholeexome sequencing experiments (WES)

\section{METHOD SUMMARY}

We describe a protocol modification of the TaqMan allelic discrimination assay (Life Technologies) for variants that reside in regions of disproportionately high GC content (>75\%). The first step of the procedure is PCR amplification of the target region with unlabeled primers. The amplified product is then used as a template for the standard primer plus probe assay. The result is improved discrimination of fluorescent signal from reference and alternate alleles, allowing genotype calls to be made. 
performed at The Centre for Applied Genomics (TCAG) (www.tcag.ca) in Toronto, Canada. A fourth variant previously genotyped in our center (7) by the standard TaqMan protocol was selected to be re-genotyped using the standard and modified protocols to test data concordance (all SNP variants are listed in Table 1). The experiments were performed on 50 ng of bloodderived genomic DNA extracted on the Gentra Autopure system (Qiagen, Hilden, Germany) from samples that were identified as containing the variant of interest (from the WES experiment) and other project-specific control samples obtained under informed consent. Briefly, primers and TaqMan MGB probes for target regions were designed using Primer Express Software Version 3.0 (Life
Table 1. Loci evaluated by the modified hydrolysis probe chemistry protocol.

\begin{tabular}{|c|c|c|c|c|}
\hline Gene & rsID/coordinates (hg19) & Primer/probe & Sequence $\left(5^{\prime}\right.$ to $\left.3^{\prime}\right)$ & Probe $\%$ GC \\
\hline \multirow[t]{4}{*}{ RAC2 } & \multirow[t]{4}{*}{ No rsID a/chr22:37,627,186 } & Fwd Primer & TTCTGATAAGGAAACACAGGCAGA & \\
\hline & & Rev Primer & CACTGGCCAAGGAGATTGGTA & \\
\hline & & Probe-G & TGGCCCTGCAGCC & 77 \\
\hline & & Probe-A & AGCCTGGCCCTACAG & 67 \\
\hline \multirow{4}{*}{$\begin{array}{l}\text { STRN4 I } \\
\text { FKRP }\end{array}$} & \multirow{4}{*}{$\begin{array}{l}\text { rs12984041/ } \\
\text { chr19:47,249,296 }\end{array}$} & Fwd Primer & GCCCTCAGGACACCCCTCGGA & \\
\hline & & Rev Primer & CCGCCGCCATCTTGGAGCAAT & \\
\hline & & Probe- $C$ & TCCCGCCCCCCCCCCGG & 94 \\
\hline & & Probe-G & TCCCGCCCCCCCGCCGG & 94 \\
\hline \multirow[t]{4}{*}{ SCARB2 } & \multirow[t]{4}{*}{ No rsIDa/chr4:77,135,017 } & Fwd Primer & AGGGCACATGACTGCGGCCT & \\
\hline & & Rev Primer & CAGCTCGGGAGAGTGCAGGGA & \\
\hline & & Probe-A & TTGCGGCGAGCCCGG & 80 \\
\hline & & Probe-C & TTGCGGCGCGCCCG & 86 \\
\hline \multirow[t]{4}{*}{ SHANK1 } & \multirow[t]{4}{*}{$\operatorname{chr} 19: 51,171,270^{\mathrm{b}}$} & Fwd Primer & ATGTTCTCCGCCGAGCCCTAC & \\
\hline & & Rev Primer & AAGCTGGTGAAGGCGCTGCT & \\
\hline & & Probe-A & TACGGGGCCGATAGC & 67 \\
\hline & & Probe-G & TACGGGGCCGGTAGC & 73 \\
\hline
\end{tabular}

${ }^{a}$ No rsID (dbSNP 138) is assigned to the variant at these coordinates.

${ }^{\mathrm{b}}$ SHANK1 variant discovered in a screen of autism spectrum disorder samples (7).

\section{A}

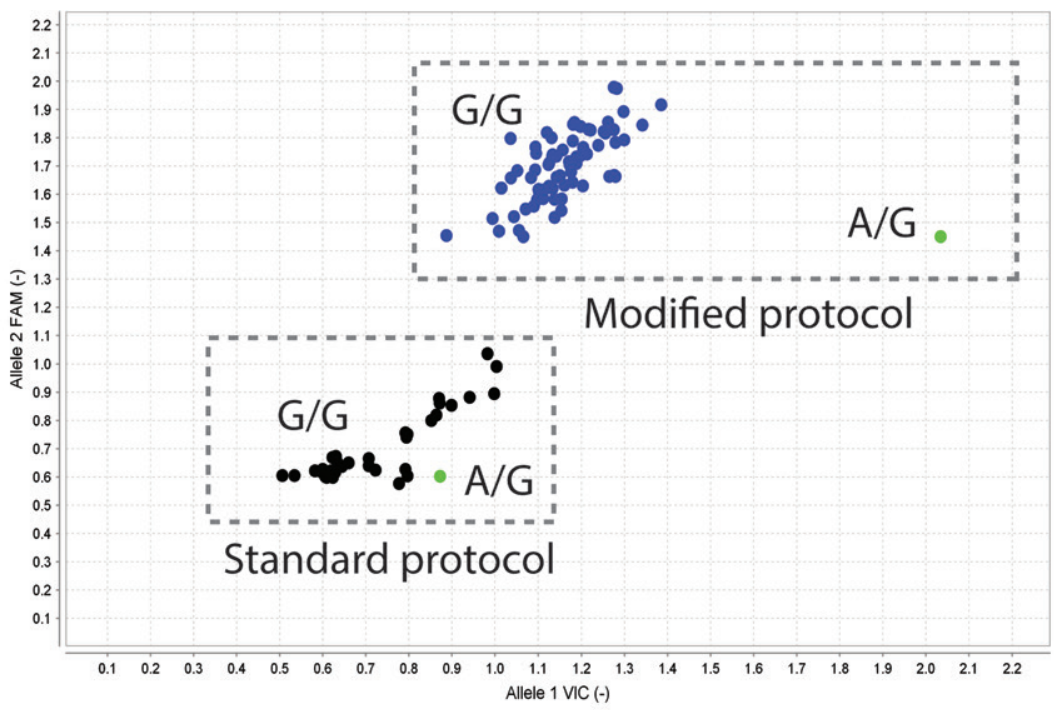

B

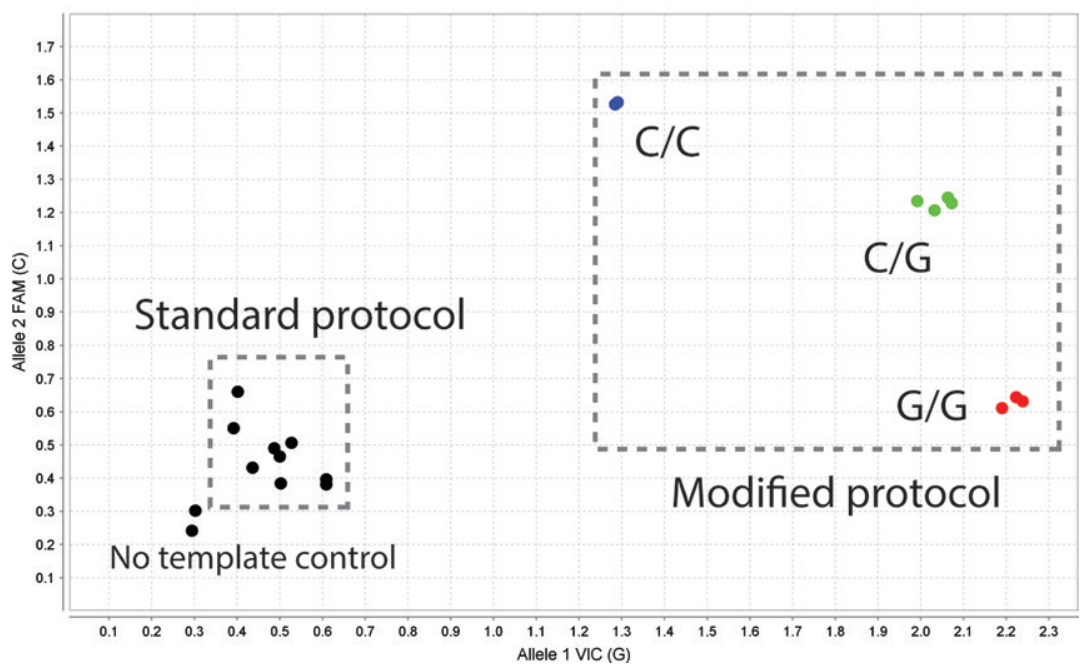

Figure 1. Plot of allele 1 (VIC) signal intensity vs. allele 2 (FAM) intensity for samples genotyped using both standard and modified protocols. (A) RAC2 variant (67\%-77\% GC) and (B) rs12984041 (94\% GC).
Technologies). PCR was performed in a $23-\mu \mathrm{L}$ reaction mixture consisting of $20 \mu \mathrm{L}$ master mix [6.9 $\mu \mathrm{L}$ water, 5.7 $\mu \mathrm{L} 5 \mathrm{M}$ betaine, $2.9 \mu \mathrm{L} 10 \times$ AmpliTaq PCR buffer, $1.4 \mu \mathrm{L} 25 \mathrm{mM} \mathrm{MgCl}_{2}$, $2.9 \mu \mathrm{L} 2 \mathrm{mM}$ dNTP mix, 1.5 units AmpliTaq polymerase (buffer, $\mathrm{MgCl}_{2}$, and AmpliTaq polymerase purchased from Life Technologies)], $1 \mu \mathrm{L}$ each of forward and reverse primer, and 1 $\mu \mathrm{L}$ genomic DNA (50 ng). The cycling conditions were: an initial denaturation step at $95^{\circ} \mathrm{C}$ for $5 \mathrm{~min}$ followed by 30 cycles of $94^{\circ} \mathrm{C}(40 \mathrm{~s}), 62^{\circ} \mathrm{C}(40 \mathrm{~s}), 72^{\circ} \mathrm{C}$ (40 s), a final extension at $72^{\circ} \mathrm{C}$ for 5 min, and hold at $4^{\circ} \mathrm{C}$ in a PTC-225 thermal cycler (Bio-Rad Laboratories, Hercules, CA). A volume of $1 \mu \mathrm{L}$ of this unpurified $P C R$ reaction was then used as input template in the standard TaqMan genotyping protocol (using the TaqMan Genotyping Mastermix, catalog number 4371357) as per the manufacturer's recommendations. End point scanning was done on the Applied Biosystems ViiA 7 Real-Time PCR System (Life Technologies). For one SNP variant, samples of each genotype (homozygous reference, heterozygous variant, and homozygous variant) were Sanger sequence validated using a 3730XL DNA Analyzer (Life Technologies). Lastly, for the SNP variant with the highest probe GC content (rs12984041), we performed the experiment with 50,40,30,20, 10, and 5 ng of input DNA for 2 samples from 


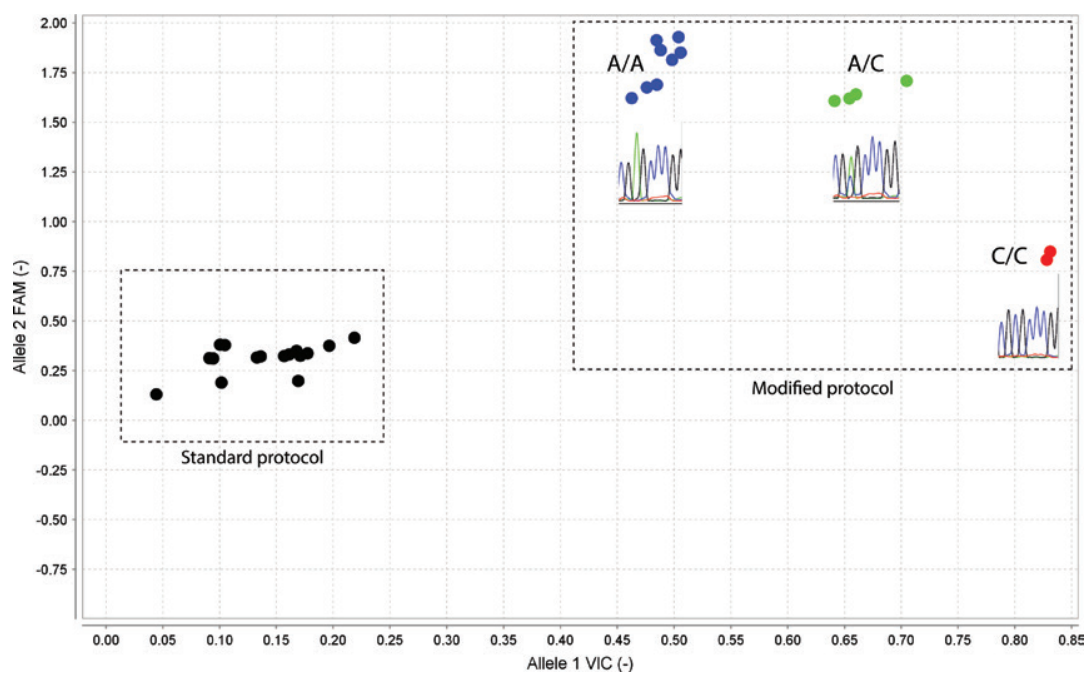

Figure 2. SCARB2 variant $(80 \%-86 \% \mathrm{GC})$ genotyped using standard versus modified protocols. Sanger sequence verification was performed for a sample from each genotype group.

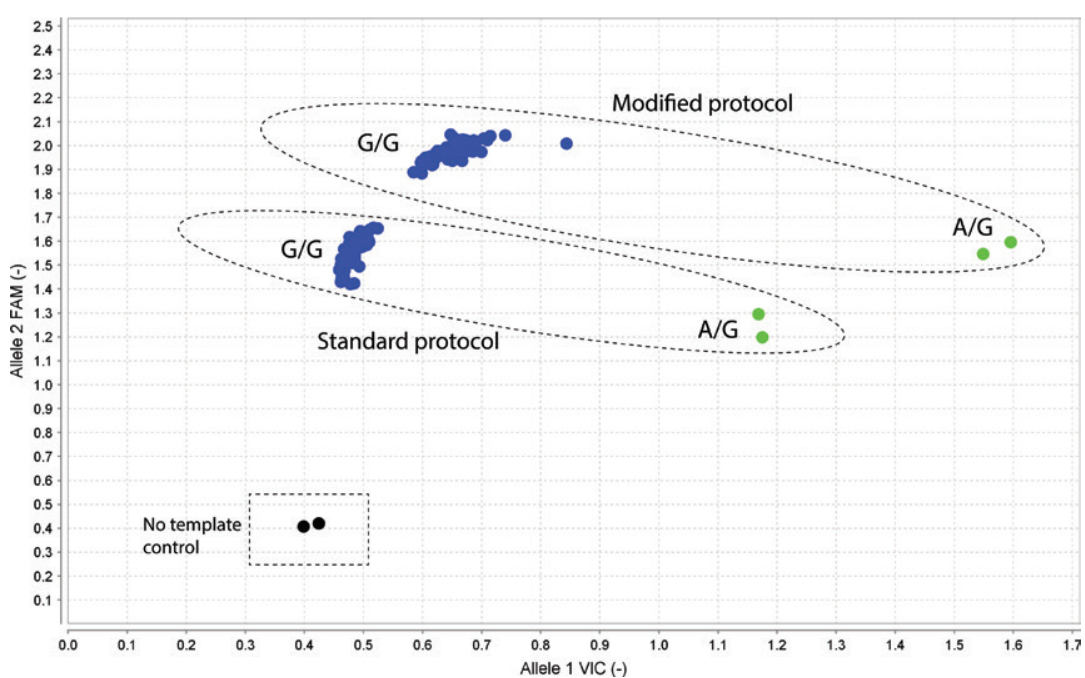

Figure 3. Test of genotype data concordance for standard versus modified protocols on a SHANK1 variant $(67 \%-73 \% \mathrm{GC})$.

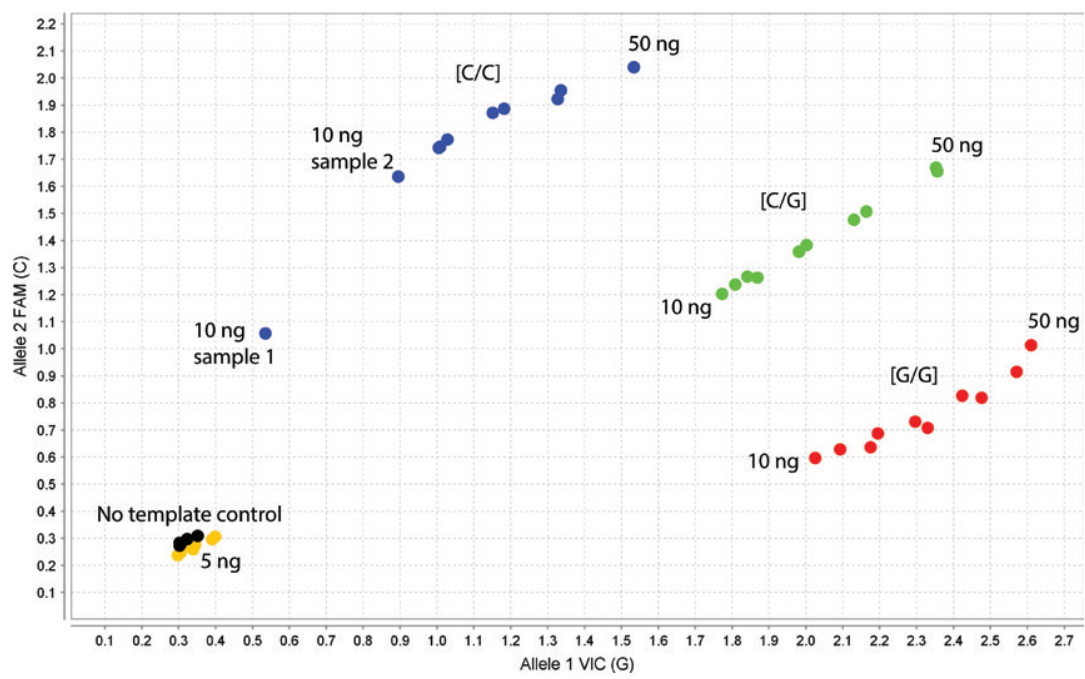

Figure 4. Two samples of each genotype group (CC, CG, and GG) were genotyped for rs 12984041. Samples selected from Figure 1B were genotyped using 50, 40, 30, 20, 10, and 5 ng of input DNA each genotype group (Figure 1B) to test the limits of the method on smaller quantities of DNA.

Genotyping data quality for the four single-nucleotide variants using the modified protocol was significantly improved over the standard manufacturer's protocol (without the pre-PCR step). The increase in signal intensity from both FAM and VIC probes results in significantly better separation of genotype clusters in the data plot (Figures 1 and 2), thus allowing a confident genotype call to be made. The modified protocol was successful for an oligonucleotide probe of $67 \%-77 \%$ GC content (Figure $1 \mathrm{~A})$, as well as a probe of $94 \% \mathrm{GC}$ content (Figure 1B). Genotyping the same samples without the pre-PCR step resulted in a merged cluster in which individual genotypes cannot be resolved (Figure 1, A or B, bottom left). We further Sanger sequenced individuals from each putative genotype group for the third variant $(80 \%-86 \%$ GC content, Figure 2) to verify that the clustering of samples was due to genuine sequence differences. To

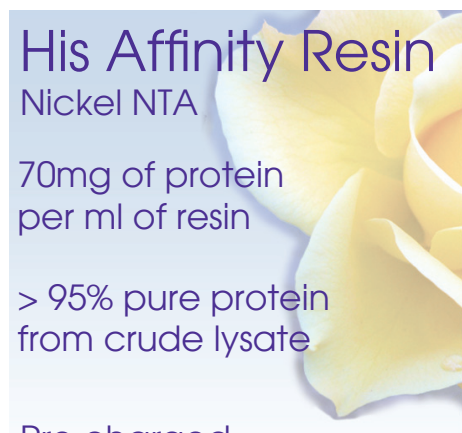

Pre charged

Ready to use matrices for any scale production

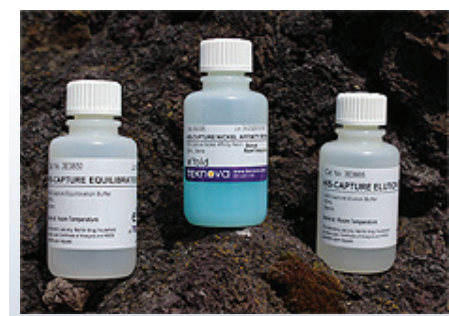

Higher Yield! Lower Price!

For more info visit: www.teknova.com/his-tag phone: 1-855-242-0011 WWW.rekrnova.com

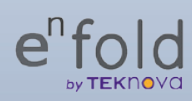




\section{BLOW UP THE HOLDUPS IN YOUR RNA RESEARCH}

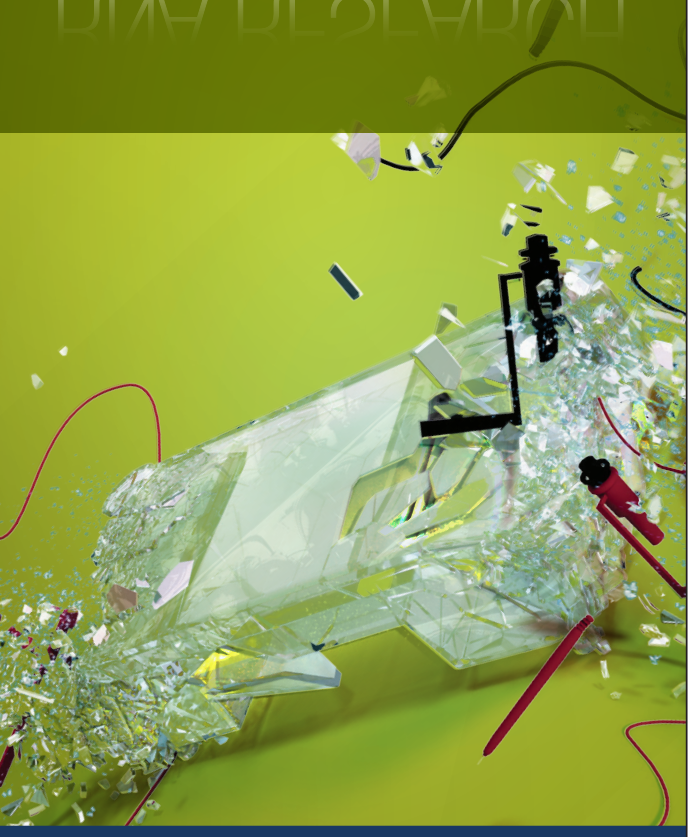

Assess RNA quality and quantity simultaneously.

\section{FULLY AUTOMATED FRAGMENT ANALYZER ${ }^{\text {TM }}$ DOES IT ALL.}

\section{Quantifies with precision (10\% CV) and accuracy $( \pm 20 \%)$}

Provides an RNA quality number that correlates to legacy data

Observes gDNA contamination in RNA

Calculates the Illumina DV 200 metric for FFPE samples

No chips. No tapes. No compromises.

More at AATI-US.COM

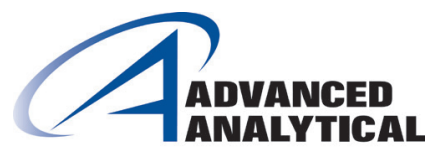

investigate whether the pre-PCR step would introduce allele-specific amplification bias, we performed the modified protocol on a SNP for which we already had data using the standard protocol (Figure 3). The genotyping data were concordant between the two experiments. Finally, we tested the method on input DNA ranging from 5 to $50 \mathrm{ng}$. Amplification was achieved for $10 \mathrm{ng}$ and higher of input template (Figure 4), but there was no amplification with 5 ng of template.

In conclusion, the results presented here show that a pre-PCR step significantly improves the performance of allelic discriminatory probe-based assays for variants in GC-rich regions. Furthermore, in the assays we have examined with our new approach, the pre-PCR step does not introduce allele-specific bias. This protocol modification will enable the genotyping of variants that were previously not amenable to this technology, thus expanding capacity for genotypic characterization.

\section{Author contributions}

A.M. conceived of the study and carried out the molecular experiments. T.A.P. drafted the manuscript, and S.W.S. edited and approved the final version.

\section{Acknowledgments}

We thank Peter Ray and Vilma Navickiene from the Department of Paediatric Laboratory Medicine at The Hospital for Sick Children for an aliquot of the assay for rs 12984041 for this study. This project was supported by funds from Genome Canada and the Ontario Genomics Institute to The Centre for Applied Genomics (a Genome Canada Science, Technology and Innovation Centre) to S.W.S. Support was also obtained from the GlaxoSmithKline-CIHR Endowed Chair in Genome Sciences to S.W.S.

\section{Competing interests}

The authors declare no competing interests.

\section{References}

1. Kalari, K.R., M. Casavant, T.B. Bair, H.L. Keen, J.M. Comeron, T.L. Casavant, and T.E. Scheetz. 2006. First exons and introns - a survey of GC content and gene structure in the human genome. In Silico Biol. 6:237-242.

2. Zhu, L., Y. Zhang, W. Zhang, S. Yang, J.Q. Chen, and D. Tian. 2009. Patterns of exon-intron architecture variation of genes in eukaryotic genomes. BMC Genomics 10:47.

3. McDowell, D.G., N.A. Burns, and H.C. Parkes. 1998. Localised sequence regions possessing high melting temperatures prevent the ampli fi cation of a DNA mimic in competitive PCR. Nucleic Acids Res. 26:3340-3347.

4. Tan, M.H., J. Gécz, and C. Shoubridge. 2013. PCR amplification and sequence analysis of GC-rich sequences: Aristalessrelated homeobox example, p. 105-120. In D.M. Hatters, and A.J. Hannan (Eds.), Tandem Repeats in Genes, Proteins, and Disease, Methods and Protocols, Series: Methods in Molecular Biology, Vol. 1017. Springer Science+Business Media Publishing, New York.

5. Livak, K.J. 1999. Allelic discrimination using fluorogenic probes and the $5^{\prime}$ nuclease assay. Genet. Anal. 14:143-149.

6. McGuigan, F.E. and S.H. Ralston. 2002. Single nucleotide polymorphism detection: allelic discrimination using TaqMan. Psychiatr. Genet. 12:133-136.

7. Sato, D., A.C. Lionel, C.S. Leblond, A. Prasad, D. Pinto, S. Walker, I. O'Connor, C. Russell, et al. 2012. SHANK1 Deletions in Males with Autism Spectrum Disorder. Am. J. Hum. Genet. 90:879-887.

Received 9 July 2014; accepted 2 October 2014.

Address correspondence to Tara A. Paton, The Centre for Applied Genomics, The Hospital for Sick Children, Toronto, Canada. E-mail: tpaton@sickkids.ca

To purchase reprints of this article, contact: biotechniques@fosterprinting.com 